\title{
COMPARATIVE STUDY OF TCM, TTCM, BICM AND BICM-ID SCHEMES
}

\author{
S. X. Ng, T. H. Liew, L-L. Yang, ${ }^{1}$ L. Hanzo \\ Dept. of Electronics and Computer Science, \\ University of Southampton, SO17 1BJ, UK. \\ Tel: +44-23-8059 3125, Fax: +44-23-80594508 \\ Email:11h@ecs.soton.ac.uk \\ http://www-mobile.ecs.soton.ac.uk
}

\begin{abstract}
Coded modulation is a bandwidth efficient scheme that combines the functions of coding and modulation. In this contribution, a comparative study of Trellis Coded Modulation (TCM), Turbo Trellis Coded Modulation (TTCM), Bit-Interleaved Coded Modulation (BICM) and Iterative Decoding assisted BICM (BICM-ID) schemes over Gaussian and uncorrelated narrowband Rayleigh fading channels is presented in the context of 8-level Phase Shift Keying (8PSK), 16-level Quadrature Amplitude Modulation (16QAM) and 64QAM. We comparatively study the associated decoding complexity, block length and bandwidth efficiency. It is shown that TTCM constitutes the best compromise scheme, followed by BICM-ID.
\end{abstract}

\section{INTRODUCTION}

One of the most important objectives in the design of digital cellular systems is the efficient exploitation of the available spectrum in order to accommodate the ever-increasing traffic demands. The design of coded modulation schemes is affected by a variety of criteria. A high squared Free Euclidean Distance (FED) is desired for Additive White Gaussian Noise (AWGN) channels, while high Effective Code Length (ECL) and minimum product distance are desired for fading channels [1].

Trellis Coded Modulation (TCM) [2] was proposed originally for Gaussian channels, which was further developed for applications in mobile communications [1, 3]. Turbo Trellis Coded Modulation (TTCM) [4] is a more recent joint coding and modulation scheme that has a structure similar to that of the family of power efficient binary turbo codes [5], but employs TCM schemes as component codes. TTCM [4] requires $0.5 d B$ lower Signal-to-Noise Ratio (SNR) at a bit error rate (BER) of $10^{-4}$, than binary turbo coded 8PSK over AWGN channels. TCM and TTCM invoked SetPartitioning (SP) based signal labeling, in order to achieve a higher FED between the unprotected bits of the constellation, so that parallel trellis transitions can be associated with the unprotected Information Bits (IBs). This reduced the decoding complexity. Furthermore, in our TCM and TTCM investigations, random symbol interleavers were utilised.

Another coded modulation scheme distinguishing itself by utilising bit-based interleaving in conjunction with Gray

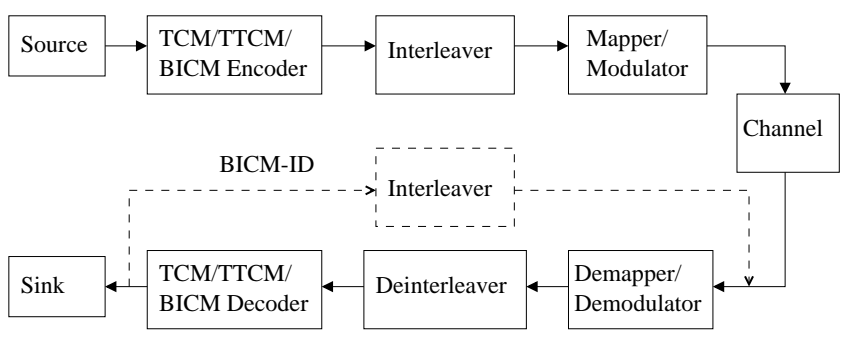

Figure 1: System overview of different coded modulation schemes.

signal constellation labeling is referred to as Bit-Interleaved Coded Modulation (BICM) [6]. More explicitly, BICM combines conventional convolutional codes with several independent bit interleavers, in order to increase the achievable diversity order. With the aid of bit interleavers the code's diversity order can be increased to the binary Hamming distance of a code [6]. The number of parallel bit-interleavers equals the number of coded bits in a symbol for the BICM scheme proposed in [6]. The performance of BICM is better than that of TCM over uncorrelated (or perfectly interleaved) narrowband Rayleigh fading channels, but worse than that of TCM in Gaussian channels due to the reduced Euclidean distance of the bit-interleaved scheme [6].

Recently iterative joint decoding and demodulation assisted BICM referred to as BICM-ID was proposed in [7, 8], which uses SP based signal labeling. The approach of BICM-ID is to increase the Euclidean distance of BICM and hence to exploit the full advantage of bit interleaving with the aid of soft-decision feedback based iterative decoding [8].

\section{SYSTEM OVERVIEW}

The schematic of the coded modulation schemes under consideration is shown in Figure 1. The source generates random IBs, which are encoded by one of the TCM, TTCM or BICM encoders. The coded sequence is then appropriately interleaved and used to modulate the waveforms according to the symbol mapping rules. For a narrowband Rayleigh fading channel in conjunction with coherent detection, the relationship between the transmitted discrete time signal $x_{t}$ and the received discrete time signal $y_{t}$ is given by

$$
y_{t}=\rho_{t} x_{t}+n_{t},
$$


where $\rho_{t}$ is the Rayleigh-distributed fading amplitude having an expected value of $\mathrm{E}\left(\rho_{t}^{2}\right)=1$, while $n_{t}$ is the complex AWGN with variance $\sigma_{I}^{2}=\sigma_{Q}^{2}=N_{0} / 2$. For AWGN channels we have $\rho_{t}=1$. The receiver consists of a coherent demodulator followed by a deinterleaver and one of the TCM, TTCM or BICM decoders. TTCM schemes consist of two component TCM encoders and two parallel decoders. In BICM-ID schemes the decoder output is appropriately interleaved and fed back to the demodulator input, as shown in Figure 1.

The log-domain branch metric required for the maximumlikelihood decoding of TCM and TTCM over fading channels is

$$
\pi_{t}=\left|y_{t}-\rho_{t} x_{t}\right|^{2},
$$

whereas the corresponding branch metric for BICM and BICM-ID is formed by summing the deinterleaved bit metrics $\lambda$ of each coded bit $v_{t}^{i}$, yielding

$$
\pi_{t}=\sum_{i=1}^{m+1} \lambda\left(v_{t}^{i}=b\right),
$$

where $i$ is the bit position of the coded bit in a constellation symbol, $m$ is the number of IBs per symbol and $b \in(0,1)$. The number of coded bits per symbol is $(m+1)$, since the coded modulation schemes add one parity bit to the $m$ IBs by doubling the original constellation size, in order to maintain the same spectral efficiency of $m$ bits/s/Hz.

The BICM bit metrics $\tilde{\lambda}$ before the deinterleaver are defined as [7]

$$
\begin{aligned}
\tilde{\lambda}\left(v_{t}^{i}=b\right) \approx & \min \left|y_{t}-\rho_{t} x\right|^{2}, \\
& x \in \chi(i, b)
\end{aligned}
$$

where $\chi(i, b)$ is the signal set, for which bit $i$ of the symbol has a binary value $b$. The average bit error probability of TCM using MPSK [2] over Rician channels at high SNR is given by [1]

$$
P_{b} \cong \frac{1}{B} C\left(\frac{(1+K) e^{-K}}{E_{s} / N_{0}}\right)^{L} ; E_{s} / N_{0} \gg K,
$$

where $C$ is a constant that depends on the distance structure of the code, $B$ is the number of bits in the transmission block, while $K$ is the Rician fading parameter. Furthermore, $L$ is the so-called shortest error event path, i.e. the ECL or the minimum symbol Hamming distance between the transmitted and received sequence. Finally, $E_{s} / N_{0}$ is the channel's symbol energy to noise spectral density ratio. It is clear from Equation 5 that $P_{b}$ varies inversely proportionally with $\left(E_{s} / N_{0}\right)^{L}$ and this ratio can be increased by time and space diversity [1]. Diversity may be obtained by repetition coding (which reduces the effective data rate), space-time coded multiple transmitter/receiver structures (which increases cost and complexity) or by simple interleaving (which induces latency).

The coded modulation schemes that we comparatively studied are Ungerboeck's TCM [2], Robertson's TTCM [4], Zehavi's BICM [6] and Li's BICM-ID [8]. Table 1 shows the generator polynomials for the TCM and TTCM codes in octal format. These are systematic codes that add one parity bit to the IBs. Hence, the coding rate for a $2^{m+1}$ ary signal in PSK or QAM is $R=\frac{m}{m+1}$. The number of

\begin{tabular}{|c|l||c|c|c|c|c|}
\hline Rate & State & $\tilde{m}$ & $H^{0}$ & $H^{1}$ & $H^{2}$ & $H^{3}$ \\
\hline \hline $2 / 3$ & 8 & 2 & 11 & 02 & 04 & - \\
$(8 \mathrm{PSK})$ & $64 *$ & 2 & 103 & 30 & 66 & - \\
\hline $3 / 4$ & 8 & 3 & 11 & 02 & 04 & 10 \\
$(16 \mathrm{QAM})$ & $64 *$ & 3 & 101 & 16 & 64 & - \\
\hline $5 / 6$ & 8 & 2 & 11 & 02 & 04 & - \\
$(64 \mathrm{QAM})$ & $64 *$ & 2 & 101 & 16 & 64 & - \\
\hline
\end{tabular}

Table 1: "Punctured" TCM codes with best minimum distance for PSK and QAM, (CRobertson and Wörz [4]. '*' indicates Ungerboeck's TCM codes [2]. Two-dimensional (2D) modulation is utilised. Octal format is used to represent the generator polynomials $H^{i}$ and $\tilde{m}$ denotes the number of coded IBs.

decoding states for a code of memory $K$ is $2^{K}$. When the number of protected IBs $\tilde{m}$ is less than the total number of

\begin{tabular}{|c|c|c|c|c|c|c|}
\hline Rate & State & $g^{1}$ & $g^{2}$ & $g^{3}$ & $g^{4}$ & $d_{\text {free }}$ \\
\hline \multirow{6}{*}{$\begin{array}{c}2 / 3 \\
(8 \mathrm{PSK})\end{array}$} & 8 & 4 & 2 & 6 & - & \multirow[t]{2}{*}{4} \\
\hline & $(M=3)$ & 1 & 4 & 7 & - & \\
\hline & 16 & 7 & 1 & 4 & - & \multirow[t]{2}{*}{5} \\
\hline & $(M=4)$ & 2 & 5 & 7 & - & \\
\hline & 64 & 15 & 6 & 15 & - & \multirow[t]{2}{*}{7} \\
\hline & $(M=6)$ & 6 & 15 & 17 & - & \\
\hline \multirow{6}{*}{$\begin{array}{c}3 / 4 \\
(16 \mathrm{QAM})\end{array}$} & 8 & 4 & 4 & 4 & 4 & \multirow[t]{3}{*}{4} \\
\hline & \multirow[t]{2}{*}{$(M=3)$} & 0 & 6 & 2 & 4 & \\
\hline & & 0 & 2 & 5 & 5 & \\
\hline & \multirow{3}{*}{$\begin{array}{c}32 \\
(M=5)\end{array}$} & 6 & 2 & 2 & 6 & \multirow[t]{3}{*}{5} \\
\hline & & 1 & 6 & 0 & 7 & \\
\hline & & 0 & 2 & 5 & 5 & \\
\hline Rate & State & $g^{1}$ & $\overline{g^{2}}$ & $\overline{P \text { Pun }}$ & turin & $\overline{d_{\text {free }}}$ \\
\hline \multirow{3}{*}{$\begin{array}{c}5 / 6 \\
(64 \mathrm{QAM})\end{array}$} & \multirow{2}{*}{$\begin{array}{c}8 \\
(M=3)\end{array}$} & \multirow[t]{2}{*}{$\overline{15}$} & \multirow[t]{2}{*}{$\overline{\overline{17}}$} & & $\overline{0110}$ & \multirow[t]{2}{*}{ 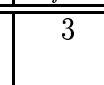 } \\
\hline & & & & & $\begin{array}{lll}111 \\
\end{array}$ & \\
\hline & $\begin{array}{c}64 \\
(M=6)\end{array}$ & 133 & 171 & & $\begin{array}{lll}1 & 1 & 1 \\
0 & 0 & 0\end{array}$ & 3 \\
\hline
\end{tabular}
original IBs $m$, there are $(m-\tilde{m})$ uncoded IBs and $2^{m-\tilde{m}}$ parallel transitions in the trellis of the code. Parallel transitions assist in reducing the decoding complexity and the memory required.

Table 2: Top table shows the generator polynomials of Paaske's code, page 331 of [9]. Bottom table shows those of the Rate-Compatible Puncture Convolutional codes [10]. $M$ is the code memory and $d_{\text {free }}$ is the free Hamming distance. Octal format is used for the polynomial coefficients $g^{i}$, while ' 1 ' and ' 0 ' in the puncturing matrix indicate the position of the unpunctured and punctured coded bits, respectively.

Table 2 shows the generator polynomials for the BICM and BICM-ID codes in octal format. These codes are nonsystematic convolutional codes having maximum free Hamming distance. Again, only one extra bit is added to the IBs. Hence, the coding rate and the spectral efficiency are similar to that of TCM and TTCM for the $2^{m+1}$-ary modulation schemes used. In order to reduce the required decoding memory, the BICM and BICM-ID schemes based on $64 \mathrm{QAM}$ were obtained by puncturing the rate- $1 / 2$ codes following the approach of [10].

Soft decision trellis decoding utilizing the Log-Maximum A Posteriori (Log-MAP) algorithm [11] was invoked for the 
decoding of the coded modulation schemes. The Log-MAP algorithm is a numerically stable version of the MAP algorithm operating in the log-domain, in order to reduce its complexity and to mitigate the numerical problems associated with the MAP algorithm [12].

\section{SIMULATION RESULTS AND DISCUSSIONS}

In this section we study the performance of TCM, TTCM, BICM and BICM-ID using computer simulations. The complexity of the coded modulation schemes is compared in terms of the number of decoding states and the number of decoding iterations. For a TCM or BICM code of memory $M$, the corresponding complexity is proportional to the number of decoding states $S=2^{M}$. Since TTCM schemes invoke two component TCM codes, a TTCM code invoking $t$ iterations and using an $S$-state component code exhibits a complexity proportional to $2 \cdot t \cdot S$ or $t \cdot 2^{M+1}$. As for BICMID schemes, only one decoder is used but the demodulator is invoked in each decoding iteration. However, the complexity of the demodulator is assumed to be insignificant compared to that of the decoder. Hence, a BICM-ID code with $t$ iterations using an $S$-state code exhibits a complexity proportional to $t \cdot S$ or $t \cdot 2^{M}$.

\subsection{AWGN Channels}

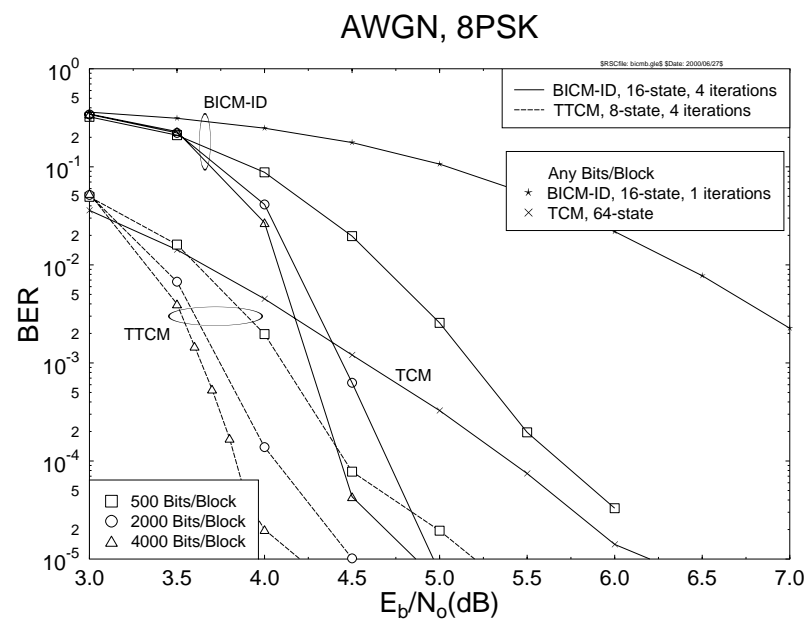

Figure 2: Effects of block length on the TCM, TTCM and BICM-ID performance in the context of an 8PSK scheme over AWGN channels.

Figure 2 illustrates the effects of interleaving block length on the TCM, TTCM and BICM-ID performance in an 8PSK scheme over AWGN channels. It is clear from the figure that a high interleaving block length is desired for the iterative TTCM and BICM-ID schemes. The block length does not affect the BICM-ID performance during the first iteration, since it constitutes a BICM scheme using SP based phasor labeling. However, if we consider four iterations, the performance improves, converging faster to the Error-FreeFeedback (EFF) bound [7] for larger block lengths. At a BER of $10^{-4}$ a 500 -bit block length was about $1 d B$ inferior in SNR terms in comparison to the 2000-bit block length in the context of the BICM-ID scheme. A slight further improvement was obtained for the 4000-bit block length. In other words, the advantage of BICM-ID over TCM is more significant for larger block lengths. The 8-state TTCM performance also improves, when using four iterations, as the block length is increased.

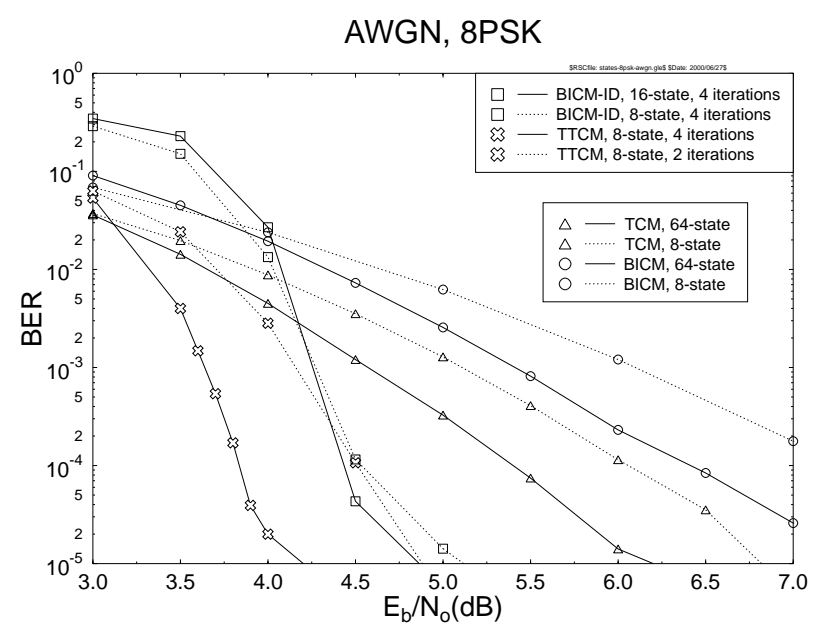

Figure 3: Effects of decoding complexity on the TCM, TTCM, BICM and BICM-ID performance in an 8PSK scheme over AWGN channels using a block length of 4000 IBs (2000 symbols).

Figure 3 shows the effects of the decoding complexity on the TCM, TTCM, BICM and BICM-ID schemes' performance in the context of an 8PSK scheme over AWGN channels using a block length of 4000 IBs (2000 symbols). The 64-state TCM, 64-state BICM, 8-state TTCM using four iterations and 16-state BICM-ID along with four iterations exhibit a similar complexity. At a BER of $10^{-4}$, TTCM requires about $0.6 d B$ lower SNR than BICM-ID, $1.6 d B$ less energy than TCM and $2.5 d B$ lower SNR than $\mathrm{BICM}$. When the coding complexity is reduced such that 8-state codes are used in the TCM, BICM and BICM-ID schemes, their corresponding performance becomes worse than that of the 64-state codes, as shown in Figure 3. In order to be able to compare the associated performance with that of 8-state BICM-ID using four iterations, 8-state TTCM along with two iterations is employed. Observe that due to the insufficient number of iterations, TTCM exhibits only marginal advantage over BICM-ID.

Figure 4 shows the performance of TCM, TTCM and BICM-ID invoking 16QAM over AWGN channels using a block length of 6000 IBs (2000 symbols). Upon comparing 64-state TCM with 32-state BICM-ID using two iterations, we observed that BICM-ID outperforms TCM, for $E_{b} / N_{0}$ in excess of $6.8 \mathrm{~dB}$. However, 8-state BICM-ID using an increased number of iterations, such as four or eight, outperforms the similar complexity 32 -state BICM-ID employing two iterations as well as 64-state TCM. About $1.2 d B$ $E_{b} / N_{0}$ gain was obtained at BER $=10^{-4}$ for 8 -state BICMID using eight iterations over 64 -state TCM at a similar decoding complexity. Comparing 8-state TTCM using two iterations and 8-state BICM-ID employing four iterations reveals that BICM-ID performs better for the $E_{b} / N_{0}$ range of $5.7 d B$ to $7 d B$. When the number of iterations is in- 


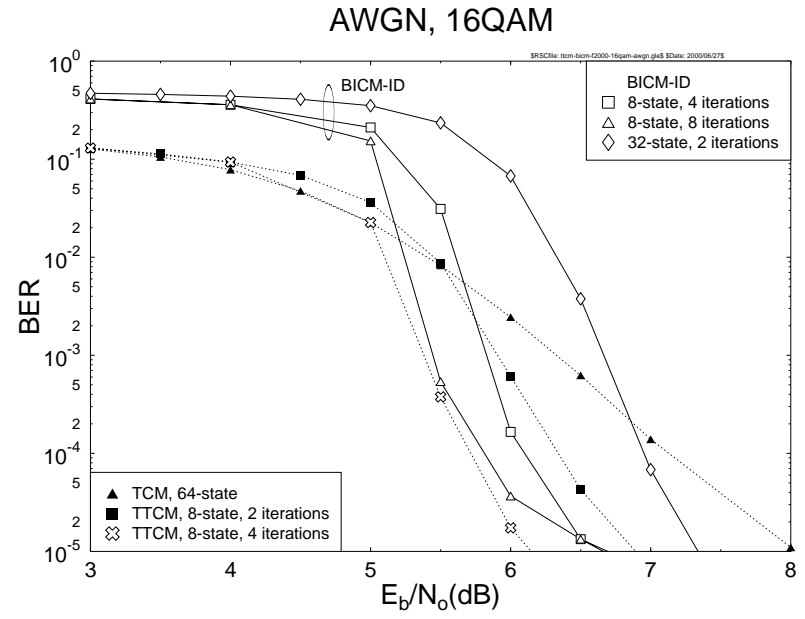

Figure 4: Comparison of TCM, TTCM and BICM-ID employing 16QAM over AWGN channels using a block length of 6000 IBs (2000 symbols).

creased to four for TTCM and to eight for BICM-ID, TTCM exhibits a better performance, as seen in Figure 4.

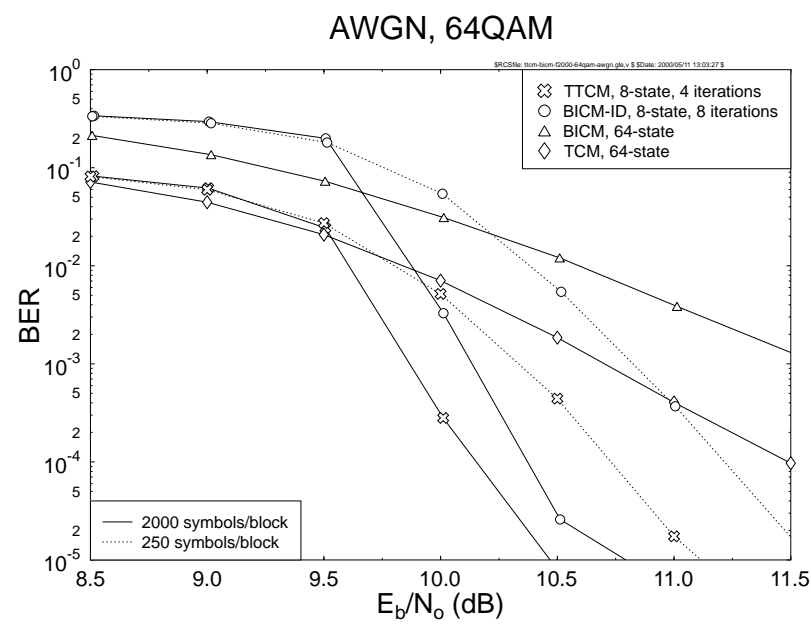

Figure 5: Comparison of TCM, TTCM, BICM and BICMID using 64QAM over AWGN channels.

Due to the associated SP, the intrasubset distance of TCM and TTCM increases down the partition tree. It was shown in [4] that we only need to encode $\tilde{m}=2$ out of 5 IBs in the 64QAM/TTCM for target BERs around $10^{-5}$ in AWGN channels. Hence there are $2^{5-\tilde{m}}=8$ parallel transitions due to the three uncoded IBs in the trellis of 64QAM/TTCM. Figure 5 illustrates the performance of TCM, TTCM, BICM and BICM-ID using 64QAM over AWGN channels. When using a block length of 10000 IBs (2000 symbols), 8-state TTCM with four iterations is the best candidate, followed by the similar complexity 8-state BICM-ID scheme employing eight iterations. Again, TCM performs better than BICM in AWGN channels. When a block length of 1250 IBs (250 symbols) was used, both TTCM and BICM-ID experienced a performance degradation. It is also seen in Figure 5 that BICM-ID performs closer to TTCM, when a longer block length is used.

\subsection{Uncorrelated Narrowband Rayleigh Fading Chan-} nels

\section{Rayleigh Fading, 8PSK}

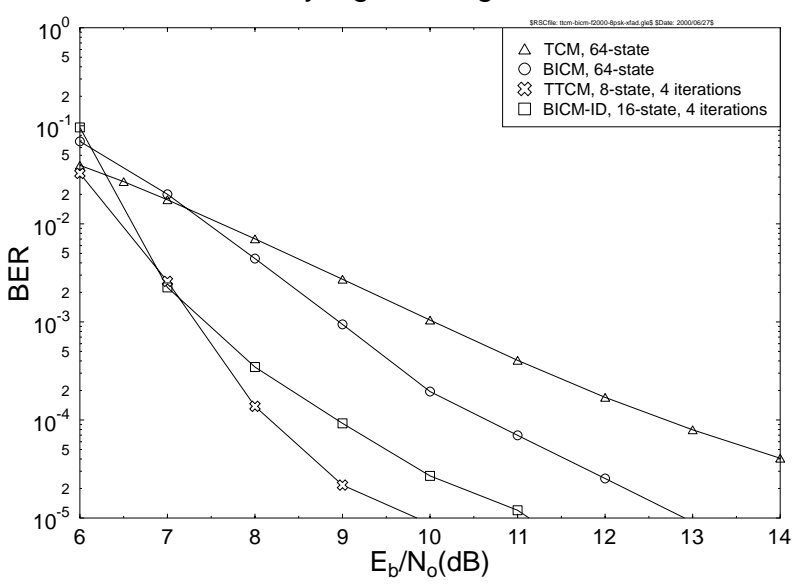

Figure 6: Comparison of TCM, TTCM, BICM and BICMID for 8PSK over uncorrelated Rayleigh fading channels using a block length of 4000 IBs (2000 symbols).

The uncorrelated Rayleigh fading channels implied using an infinite-length interleaver over narrowband Rayleigh fading channels. Figure 6 shows the performance of 64state TCM, 64-state BICM, 8-state TTCM using four iterations and 16-state BICM-ID employing four iterations in an 8PSK scheme over uncorrelated narrowband Rayleigh fading channels for a block length of 4000 IBs (2000 symbols). These four coded modulation schemes have a similar complexity. As can be seen from Figure 6, TTCM performs best, followed by BICM-ID, BICM and TCM. At a BER of $10^{-4}$, TTCM performs about $0.7 d B$ better than BICMID, $2.3 d B$ better than BICM and $4.5 d B$ better than TCM. The error floor of TTCM [4] was lower than the associated EFF bound of BICM-ID. However, the BERs of TTCM and BICM-ID were identical at $E_{b} / N_{0}=7 d B$.

Figure 7 compares the performance of TCM, TTCM and BICM-ID invoking 16QAM over uncorrelated narrowband Rayleigh fading channels using a block length of 6000 IBs (2000 symbols). Observe that 32-state BICM-ID using two iterations outperforms 64 -state TCM for $E_{b} / N_{0}$ in excess of $9.6 \mathrm{~dB}$. For the same complexity, the 8-state BICMID invoking eight iterations outperforms 64 -state TCM beyond $E_{b} / N_{0}=8.2 \mathrm{~dB}$. Similar to $8 \mathrm{PSK}$, the coding gain of BICM-ID over TCM in the context of 16QAM is more significant over narrowband Rayleigh fading channels compared to AWGN channels. Near the $E_{b} / N_{0}$ of $11 d B$ the 8-state BICM-ID approaches the EFF bound, hence 32state BICM-ID using two iterations exhibits a better performance due to its lower EFF bound. Observe also that 8-state BICM-ID using four iterations outperforms 8-state TTCM employing two iterations in the range of $E_{b} / N_{0}=$ $8.5 d B$ to $12.1 d B$. Increasing the number of iterations only marginally improve the BICM-ID, but results in a significant gain for TTCM. The performance of 8-state TTCM using four iterations is better than that of 8-state BICM- 
Rayleigh Fading, 16QAM

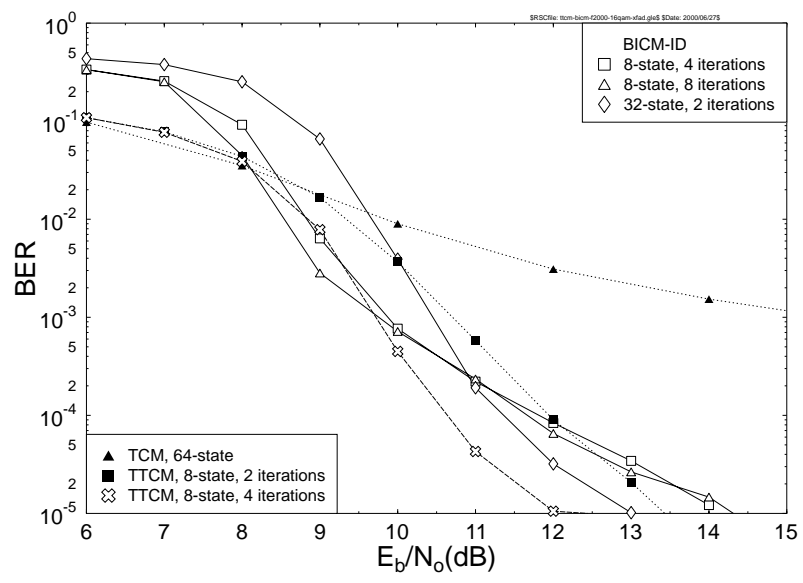

Figure 7: Comparison of TCM, TTCM and BICMID for 16QAM over uncorrelated narrowband Rayleigh fading channels transmitting 2000 symbols/block (6000 IBs/block).

ID along with eight iterations for $E_{b} / N_{0}$ values in excess of $9.6 \mathrm{~dB}$.

\section{Rayleigh Fading, 64QAM}

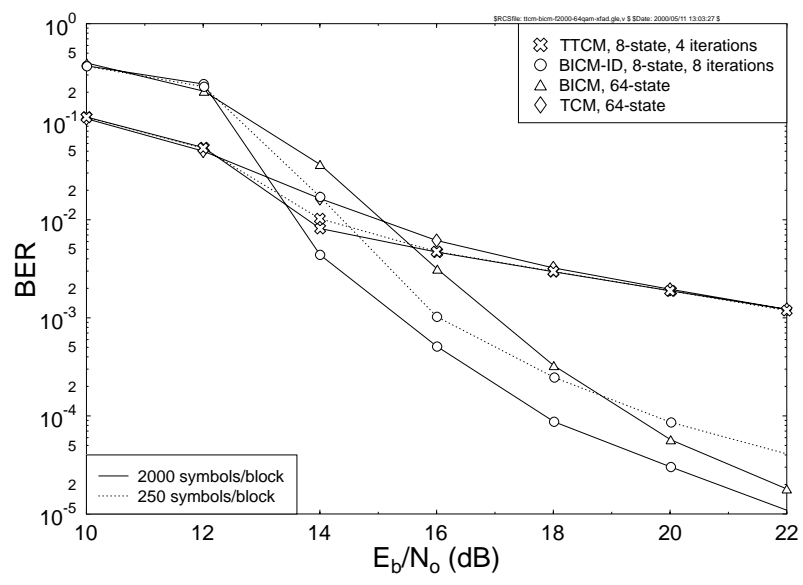

Figure 8: Comparison of TCM, TTCM, BICM and BICMID using 64QAM over uncorrelated narrowband Rayleigh fading channels.

Figure 8 illustrates the performance of TCM, TTCM, BICM and BICM-ID invoking 64QAM over uncorrelated narrowband Rayleigh fading channels. Using a block length of 10000 IBs (2000 symbols), 64-state BICM performs better than 64-state TCM for $E_{b} / N_{0}$ in excess of $15 d B$. BICMID exhibits a lower error floor than TTCM in this scenario, since BICM-ID encodes all the five IBs while TTCM encodes only two of the six 64QAM bits. The three unprotected IBs of TCM and TTCM render these schemes less robust to the uncorrelated fading channel. If we use a better TCM or TTCM code generator that encodes all the five IBs, a better performance is expected. Reducing the block length from 2000 symbols to 250 symbols resulted in a small degradation for TTCM, but yielded a significant degrada- tion for BICM-ID

\section{CONCLUSION}

In conclusion, at a given complexity $\mathrm{TCM}$ performs better than BICM in AWGN channels, but worse in uncorrelated narrowband Rayleigh fading channels. However, BICM-ID using soft decision feedback outperforms TCM and BICM over both AWGN and uncorrelated narrowband Rayleigh fading channels at the same decoding complexity. TTCM has shown superior performance over the other coded modulation schemes studied, but exhibited a higher error floor due to the uncoded IBs over uncorrelated narrowband Rayleigh fading channels.

\section{ACKNOWLEDGEMENTS}

The financial support of the European Union under the auspices of the TRUST project and the EPSRC, Swindon UK is gratefully acknowledged.

\section{REFERENCES}

[1] D. Divsalar and M. K. Simon, "The design of trellis coded MPSK for fading channel: Performance criteria," IEEE Transactions on Communications, vol. 36, pp. 1004-1012, September 1988.

[2] G. Ungerboeck, "Channel coding with multilevel/Phase signal," IEEE Transactions on Information Theory, vol. 28, pp. 55-66, January 1982.

[3] D. Divsalar and M. K. Simon, "The design of trellis coded MPSK for fading channel: Set partitioning for optimum code design," IEEE Transactions on Communications, vol. 36, pp. 1013-1021, September 1988.

[4] P. Robertson, T. Wörz, "Bandwidth-Efficient Turbo TrellisCoded Modulation Using Punctured Component Codes," IEEE Journal on Selected Areas in Communications, vol. 16, pp. 206-218, February 1998.

[5] C. Berrou, A. Glavieux and P. Thitimajshima, "Near Shannon Limit Error-Correcting Coding and Decoding : Turbo Codes," in Proceedings of IEEE International Conference on Communications, pp. 1064 - 1070, 1993.

[6] E. Zehavi, "8-PSK trellis codes for a Rayleigh fading channel," IEEE Transactions on Communications, vol. 40, pp. 873 - 883, May 1992.

[7] X. Li, J. A. Ritcey, "Trellis-Coded Modulation with Bit Interleaving and Iterative Decoding," IEEE Journal on Selected Areas in Communications, vol. 17, No. 4, April 1999.

[8] X. Li, J. A. Ritcey, "Bit-interleaved coded modulation with iterative decoding using soft feedback," IEE Electronics Letters, vol. 34, No. 10, pp. 942 - 943, May 1998.

[9] S. Lin and D. J. Costello, "Error Control Coding: Fundamentals and Applications," Englewood Cliffs, NJ: PrenticeHall, 1983.

[10] L. H. C. Lee, "New Rate-Compatible Puncture Convolutional Codes for Viterbi Decoding," IEEE Transactions on Communications, vol. 42, pp. 3073 - 3079, December 1994.

[11] P. Robertson, E. Villebrum and P. Hoeher, "A Comparison of Optimal and Sub-Optimal MAP Decoding Algorithms Operating in Log Domain," in Proceedings of the International Conference on Communications, pp. 1009-1013, June 1995.

[12] L. R. Bahl, J. Cocke, F. Jelinek and J. Raviv, "Optimal Decoding of Linear Codes for Minimizing Symbol Error Rate," IEEE Transactions on Information Theory, vol. 20, pp. 284-287, March 1974 . 